\title{
Half-arc-transitive graphs of prime-cube order of small valencies*
}

\author{
Yi Wang, Yan-Quan Feng ${ }^{\dagger}$ \\ Department of Mathematics, Beijing Jiaotong University, Beijing, P.R. China
}

Received 30 October 2015, accepted 25 November 2016, published online 20 March 2017

\begin{abstract}
A graph is called half-arc-transitive if its full automorphism group acts transitively on vertices and edges, but not on arcs. It is well known that for any prime $p$ there is no halfarc-transitive graph of order $p$ or $p^{2}$. In 1992, Xu classified half-arc-transitive graphs of order $p^{3}$ and valency 4 . In this paper we classify half-arc-transitive graphs of order $p^{3}$ and valency 6 or 8 . In particular, the first known infinite family of half-arc-transitive Cayley graphs on non-metacyclic $p$-groups is constructed.
\end{abstract}

Keywords: Cayley graph, half-arc-transitive graph, automorphism group.

Math. Subj. Class.: 05C10, 05C25, 20B25

\section{Introduction}

A (di)graph $\Gamma$ consists of a pair of sets $(V(\Gamma), E(\Gamma))$, where $V(\Gamma)$ is its vertex set, and $E(\Gamma)$ is its edge set. For a graph, $E(\Gamma)$ is also called undirected edge set and is a subset of the set $\{\{u, v\} \mid u, v \in V(\Gamma)\}$, and for a digraph, $E(\Gamma)$ is also called directed edge set and is a subset of the set $\{(u, v) \mid u, v \in V(\Gamma)\}$. For an edge $\{u, v\}$ of a graph $\Gamma$, we call $(u, v)$ an $\operatorname{arc}$ of $\Gamma$. An automorphism of a (di)graph $\Gamma$ is a permutation on $V(\Gamma)$ preserving the adjacency of $\Gamma$, and all automorphisms of $\Gamma$ form a group under the composition of permutations, called the full automorphism group of $\Gamma$ and denoted by $\operatorname{Aut}(\Gamma)$. A (di)graph $\Gamma$ is vertex-transitive or edge-transitive if $\operatorname{Aut}(\Gamma)$ acts transitively on $V(\Gamma)$ or $E(\Gamma)$, respectively. A graph $\Gamma$ is arc-transitive or symmetric if $\operatorname{Aut}(\Gamma)$ is transitive on the arc set of $\Gamma$, and half-arc-transitive provided that it is vertex-transitive, edge-transitive, but not arc-transitive. Throughout this paper, all (di)graphs $\Gamma$ are finite and simple, that is, $|V(\Gamma)|$ is finite and there are no loops or multiple edges.

*This work was supported by the National Natural Science Foundation of China $(11231008,11571035)$ and by the 111 Project of China (B16002).

${ }^{\dagger}$ Corresponding author

E-mail addresses: yiwang@bjtu.edu.cn (Yi Wang), yqfeng@bjtu.edu.cn (Yan-Quan Feng) 
Let $G$ be a permutation group on a set $\Omega$ and $\alpha \in \Omega$. Denote by $G_{\alpha}$ the stabilizer of $\alpha$ in $G$, that is, the subgroup of $G$ fixing the point $\alpha$. We say that $G$ is semiregular on $\Omega$ if $G_{\alpha}=1$ for every $\alpha \in \Omega$ and regular if $G$ is transitive and semiregular.

Let $G$ be a finite group and $S$ a subset of $G$ such that $1 \notin S$. The Cayley digraph $\Gamma=\operatorname{Cay}(G, S)$ on $G$ with respect to $S$ is defined as the digraph with vertex set $V(\Gamma)=G$ and directed edge set $\{(g, s g) \mid g \in G, s \in S\}$. The Cayley digraph Cay $(G, S)$ is connected if and only if $G=\langle S\rangle$, and if $S$ is symmetric, that is, $S^{-1}=\left\{s^{-1} \mid s \in S\right\}=S$, then $\operatorname{Cay}(G, S)$ can be viewed as a graph by identifying the two oppositely directed edges $(g, s g)$ and $(s g, g)$ as an undirected edge $\{g, s g\}$. Thus a Cayley graph can be viewed as a special case of a Cayley digraph. It is easy to see that $\operatorname{Aut}(\operatorname{Cay}(G, S))$ contains the right regular representation $\hat{G}=\{\hat{g} \mid g \in G\}$ of $G$, where $\hat{g}$ is the map on $G$ defined by $x \mapsto x g$, $x \in G$, and $\hat{G}$ is regular on the vertex set $V(\Gamma)$. This implies that a Cayley digraph is vertex-transitive. Also, it is easy to check that $\operatorname{Aut}(G, S)=\left\{\alpha \in \operatorname{Aut}(G) \mid S^{\alpha}=S\right\}$ is a subgroup of $\operatorname{Aut}(\operatorname{Cay}(G, S))_{1}$, the stabilizer of the vertex 1 in $\operatorname{Aut}(\operatorname{Cay}(G, S))$. A Cayley digraph $\operatorname{Cay}(G, S)$ is said to be normal if $\hat{G}$ is normal in $\operatorname{Aut}(\operatorname{Cay}(G, S))$.

In 1966, Tutte [26] initiated an investigation of half-arc-transitive graphs by showing that a vertex- and edge-transitive graph with odd valency must be arc-transitive. A few years later, in order to answer Tutte's question of the existence of half-arc-transitive graphs of even valency, Bouwer [5] gave a construction of a $2 k$-valent half-arc-transitive graph for every $k \geq 2$. Following these two classical articles, half-arc-transitive graphs have been extensively studied from different perspectives over decades by many authors. See, for example, $[3,13,15,18,20,23,25,33]$. One of the standard problems in the study of half-arc-transitive graphs is to classify such graphs of certain orders. Let $p$ be a prime. It is well known that there are no half-arc-transitive graphs of order $p$ or $p^{2}$, and by Cheng and Oxley [6], there are no half-arc-transitive graphs of order $2 p$. Alspach and $\mathrm{Xu}$ [2] classified half-arc-transitive graphs of order $3 p$ and Dobson [9] classified half-arc-transitive graphs of order a product of two distinct primes. Classification of half-arc-transitive graphs of order $4 p$ had been considered for more than 10 years by many authors, and recently was solved by Kutnar et al. [16]. Despite all of these efforts, however, further classifications of half-arc-transitive graphs with general valencies seem to be very difficult.

In view of the fact that 4 is the smallest admissible valency for a half-arc-transitive graph, special attention has rightly been given to the study of tetravalent half-arc-transitive graphs. In particular, constructing and classifying tetravalent half-arc-transitive graphs is currently an active topic in algebraic graph theory (for example, see [10, 11, 22, 28]). Marušič [20] and Šparl [27] classified tightly attached tetravalent half-arc-transitive graphs with odd and even radius, respectively. For quite some time, all known examples of tetravalent half-arc-transitive graphs had vertex-stabilizers that are either abelian or dihedral: For instance, Marušič [21] constructed an infinite family of tetravalent half-arc-transitive graphs having vertex stabilizers isomorphic to $\mathbb{Z}_{2}^{m}$ for each positive integer $m \geq 1$, and Conder and Marušič [7] constructed a tetravalent half-arc-transitive graph with vertexstabilizer isomorphic to $D_{4}$ of order 8 . Recently, a tetravalent half-arc-transitive graph with vertex-stabilizers that are neither abelian nor dihedral was constructed by Conder et al. [8].

$\mathrm{Xu}$ [31] classified tetravalent half-arc-transitive graphs of order $p^{3}$ for each prime $p$, and later this was extended to the case of $p^{4}$ by Feng et al. [10]. In this paper, we classify half-arc-transitive graphs of order $p^{3}$ and valency 6 or 8 . In these new constructions, there is an infinite family of half-arc-transitive Cayley graphs on non-metacyclic $p$-groups, and to our best knowledge, this is the first known construction of such graphs. 
Denote by $\mathbb{Z}_{n}$ the cyclic group of order $n$ as well as the ring of order $n$. From elementary group theory we know that up to isomorphism there are only five groups of order $p^{3}$, that is, three abelian groups $\mathbb{Z}_{p^{3}}, \mathbb{Z}_{p^{2}} \times \mathbb{Z}_{p}$ and $\mathbb{Z}_{p} \times \mathbb{Z}_{p} \times \mathbb{Z}_{p}$, and two non-abelian groups $G_{1}(p)$ and $G_{2}(p)$ defined as

$$
G_{1}(p)=\left\langle a, b \mid a^{p^{2}}=1, b^{p}=1, b^{-1} a b=a^{1+p}\right\rangle
$$

and

$$
G_{2}(p)=\left\langle a, b, c \mid a^{p}=b^{p}=c^{p}=1,[a, b]=c,[a, c]=[b, c]=1\right\rangle .
$$

It is easy to check that the center of $G_{1}(p)$ is $\left\langle a^{p}\right\rangle$ and the center of $G_{2}(p)$ is $\langle c\rangle$.

Denote by $\mathbb{Z}_{n}^{*}$ the multiplicative group of the ring $\mathbb{Z}_{n}$ consisting of numbers coprime to $n$. Let $e$ be an element of order $j<p$ in $\mathbb{Z}_{p^{2}}^{*}$. Since $\mathbb{Z}_{p^{2}}^{*} \cong \mathbb{Z}_{p(p-1)}$, we have $j \mid(p-1)$. For each $k \in \mathbb{Z}_{p}^{*}$, let $T^{j, k}=\left\{b^{k} a, b^{k} a^{e}, \ldots, b^{k} a^{e^{j-1}},\left(b^{k} a\right)^{-1},\left(b^{k} a^{e}\right)^{-1}, \ldots,\left(b^{k} a^{e^{j-1}}\right)^{-1}\right\}$ be a subset of $G_{1}(p)$ and define

$$
\Gamma^{j, k}(p)=\operatorname{Cay}\left(G_{1}(p), T^{j, k}\right) .
$$

By Proposition 2.2, $\Gamma^{j, k}(p)$ does not depend on the choice of the element $e$ of order $j$.

Suppose $4 \mid(p-1)$ and let $\lambda$ be an element of order 4 in $\mathbb{Z}_{p}^{*}$. For each $k \in \mathbb{Z}_{p}$ with $k \neq 2^{-1}(1+\lambda)$, let $S_{4, k}=\left\{a, b, a^{\lambda} b^{\lambda-1} c^{k}, a^{-\lambda-1} b^{-\lambda} c^{1-k}, a^{-1}, b^{-1},\left(a^{\lambda} b^{\lambda-1} c^{k}\right)^{-1}\right.$, $\left.\left(a^{-\lambda-1} b^{-\lambda} c^{1-k}\right)^{-1}\right\}$ be a subset of $G_{2}(p)$ and define

$$
\Gamma_{4, k}(p)=\operatorname{Cay}\left(G_{2}(p), S_{4, k}\right) .
$$

There are exactly two elements of order 4 in $\mathbb{Z}_{p}^{*}$, that is, $\lambda$ and $\lambda^{-1}=-\lambda$. Let

$$
\bar{S}_{4, s}=\left\{a, b, a^{-\lambda} b^{-\lambda-1} c^{s}, a^{\lambda-1} b^{\lambda} c^{1-s}, a^{-1}, b^{-1},\left(a^{-\lambda} b^{-\lambda-1} c^{s}\right)^{-1},\left(a^{\lambda-1} b^{\lambda} c^{1-s}\right)^{-1}\right\},
$$

where $s \in \mathbb{Z}_{p}$ and $s \neq 2^{-1}(1-\lambda)$. For each $k \in \mathbb{Z}_{p}$ and $k \neq 2^{-1}(1+\lambda)$, the automorphism of $G_{2}(p)$ induced by $a \mapsto a, b \mapsto a^{\lambda-1} b^{\lambda} c^{1-k+\lambda}, c \mapsto c^{\lambda}$, maps $S_{4, k}$ to $\bar{S}_{4, k-\lambda}$, and so $\operatorname{Cay}\left(G_{2}(p), S_{4, k}\right) \cong \operatorname{Cay}\left(G_{2}(p), \bar{S}_{4, k-\lambda}\right)$. Since $k \neq 2^{-1}(1+\lambda)$, we have $k-\lambda \neq$ $2^{-1}(1-\lambda)$. Thus, $\Gamma_{4, k}(p)$ does not depend on the choice of $\lambda$. The following is the main result of the paper.

Theorem 1.1. Let $\Gamma$ be a graph of order $p^{3}$ for a prime $p$. Then we have:

(1) If $\Gamma$ has valency 6 then $\Gamma$ is half-arc-transitive if and only if $3 \mid(p-1)$ and $\Gamma \cong$ $\Gamma^{3, k}(p)$. There are exactly $(p-1) / 2$ nonisomorphic half-arc-transitive graphs of the form $\Gamma^{3, k}(p)$;

(2) If $\Gamma$ has valency 8 then $\Gamma$ is half-arc-transitive if and only if $4 \mid(p-1)$ and $\Gamma \cong$ $\Gamma^{4, k}(p)$ or $\Gamma_{4, k}(p)$. There are exactly $p-1$ nonisomorphic half-arc-transitive graphs of the forms $\Gamma^{4, k}(p)$ and $\Gamma_{4, k}(p)$, with $(p-1) / 2$ such graphs in each form.

\section{Preliminaries}

We start by stating some group-theoretical results. For a group $G$ and $x, y \in G$, denote by $[x, y]$ the commutator $x^{-1} y^{-1} x y$ and by $x^{y}$ the conjugation $y^{-1} x y$. The following proposition is a basic property of commutators and its proof is straightforward (also see [24, Subsection 5.1.5]): 
Proposition 2.1 ([14, Kapitel III, Hilfssätze 1.2 and 1.3]). Let $G$ be a group. Then, for any $x, y, z \in G$, we have $[x, y]=[y, x]^{-1},[x y, z]=[x, z]^{y}[y, z]$ and $[x, y z]=[x, z][x, y]^{z}$. Furthermore, if $[x, y]$ commutes with $x$ and $y$ then for any integers $i$ and $j,\left[x^{i}, y^{j}\right]=$

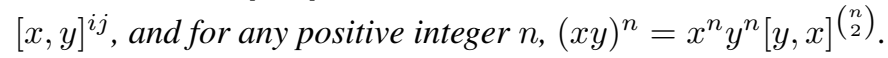

We remark that it is easy to see that the equality $(x y)^{n}=x^{n} y^{n}[y, x]^{\left(\begin{array}{l}n \\ 2\end{array}\right)}$ holds also for negative integers $n$ if we define $\left(\begin{array}{c}n \\ 2\end{array}\right)=\frac{n(n-1)}{2}$. By Li and Sim [18, Theorem 1.1 and Lemma 2.6], we have the following proposition.

Proposition 2.2. Let $\Gamma$ be a Cayley graph on $G_{1}(p)$ of valency $2 j$ with $1<j<p$. Then $\Gamma$ is half-arc-transitive if and only if $j \mid(p-1)$ and $\Gamma \cong \Gamma^{j, k}(p)$ for $1 \leq k \leq p-1$, and $\Gamma^{j, k}(p) \cong \Gamma^{j^{\prime}, k^{\prime}}(p)$ if and only if $j=j^{\prime}$ and $k=k^{\prime}(\bmod p)$. Furthermore, for each $j \mid(p-1)$ there exist exactly $(p-1) / 2$ nonisomorphic such graphs of the form $\Gamma^{j, k}(p)$.

Since a transitive permutation group of prime degree $p$ has a regular Sylow $p$-subgroup, every vertex-transitive digraph of order a prime must be a Cayley digraph. Together with the results given by Marušič [19], we have the following proposition.

Proposition 2.3. Any vertex-transitive digraph of order $p^{k}$ with $1 \leq k \leq 3$ is a Cayley digraph on a group of order $p^{k}$.

For any abelian group $H$, the map $h \mapsto h^{-1}, h \in H$ is an automorphism of $H$. By [10, Proposition 2.10], we have the following proposition.

Proposition 2.4. Let $G$ be a finite group and $\operatorname{Cay}(G, S)$ a connected half-arc-transitive Cayley graph. Then, $S$ does not contain an involution and for any $s \in S$, there is no $\alpha \in \operatorname{Aut}(G, S)$ satisfying $s^{\alpha}=s^{-1}$. Furthermore, every edge-transitive Cayley graph on an abelian group is also arc-transitive.

The following proposition is about isomorphisms between Cayley graphs on $p$-groups.

Proposition 2.5 ([17, Theorem $1.1(3)])$. Let $\operatorname{Cay}(G, S)$ and $\operatorname{Cay}(G, T)$ be two connected Cayley graphs on a p-group $G$ with respect to subsets $S$ and $T$, and let $|S|=|T|<2 p$. Then $\operatorname{Cay}(G, S)$ and $\operatorname{Cay}(G, T)$ are isomorphic if and only if there is an automorphism $\alpha$ of $G$ such that $S^{\alpha}=T$.

Let $\Gamma=\operatorname{Cay}(G, S)$ be a Cayley digraph on a finite group $G$. By Godsil [12, Lemma 2.2] (also see [32, Proposition 1.5]), we have $\mathrm{N}_{\operatorname{Aut} \Gamma}(\hat{G})=\hat{G} \rtimes \operatorname{Aut}(G, S)$.

Proposition 2.6. A Cayley digraph $\Gamma=\operatorname{Cay}(G, S)$ is normal if and only if $\operatorname{Aut}(\Gamma)_{1}=$ $\operatorname{Aut}(G, S)$.

A finite group $G$ is called 2-genetic if each normal subgroup of $G$ can be generated by two elements. For a prime $p$, denote by $\mathrm{O}_{p}(G)$ the largest normal $p$-subgroup of $G$, and by $\Phi(G)$ the Frattini subgroup of $G$, that is, the intersection of all maximal subgroups of $G$. We call $G$ a $p^{\prime}$-group if the order of $G$ is not divisible by $p$. The following proposition is about automorphism groups of Cayley digraphs on 2-genetic groups.

Proposition 2.7 ([30, Theorem 1.1]). Let $G$ be a nonabelian 2-genetic group of order $p^{n}$ for an odd prime $p$ and a positive integer $n$, and let $\Gamma=\operatorname{Cay}(G, S)$ be a connected Cayley digraph. Assume that $\operatorname{Aut}(G, S)$ is a $p^{\prime}$-group and $\Gamma$ is non-normal. Then $p \in\{3,5,7,11\}$ 
and $\operatorname{ASL}(2, p) \leq \operatorname{Aut}(\Gamma) / \Phi\left(\mathrm{O}_{p}(A)\right) \leq \operatorname{AGL}(2, p)$. Furthermore, the kernel of $A:=$ Aut $(\Gamma)$ acting on the quotient digraph $\Gamma_{\Phi\left(\mathrm{O}_{p}(A)\right)}$ is $\Phi\left(\mathrm{O}_{p}(A)\right)$, and one of the following happens:

(1) $p=3, n \geq 5$, and $\Gamma_{\Phi\left(\mathrm{O}_{p}(A)\right)}$ has out-valency at least 8 ;

(2) $p=5, n \geq 3$ and $\Gamma_{\Phi\left(\mathrm{O}_{p}(A)\right)}$ has out-valency at least 24 ;

(3) $p=7, n \geq 3$ and $\Gamma_{\Phi\left(\mathrm{O}_{p}(A)\right)}$ has out-valency at least 48 ;

(4) $p=11, n \geq 3$ and $\Gamma_{\Phi\left(\mathrm{O}_{p}(A)\right)}$ has out-valency at least 120 .

In Proposition 2.7, the quotient digraph $\Gamma_{\Phi\left(\mathrm{O}_{p}(A)\right)}$ has the orbits of $\Phi\left(\mathrm{O}_{p}(A)\right)$ on $V(\Gamma)$ as vertices, and for two orbits $O_{1}$ and $O_{2},\left(O_{1}, O_{2}\right)$ is a directed edge in $\Gamma_{\Phi\left(\mathrm{O}_{p}(A)\right)}$ if and only if $(u, v)$ is a directed edge in $\Gamma$ for some $u \in O_{1}$ and $v \in O_{2}$.

\section{Proof of Theorem 1.1}

Let $\Gamma$ be a half-arc-transitive graph and $A=\operatorname{Aut}(\Gamma)$. Let $(u, v)$ be an arc of $\Gamma$ and set $(u, v)^{A}=\left\{\left(u^{a}, v^{a}\right) \mid a \in A\right\}$. Define digraphs $\Gamma_{1}$ and $\Gamma_{2}$ having vertex set $V(\Gamma)$ and directed edge sets $(u, v)^{A}$ and $(v, u)^{A}$, respectively. Since $\Gamma$ is half-arc-transitive, for every edge $\{x, y\} \in E(\Gamma)$, each of $\Gamma_{1}$ and $\Gamma_{2}$ contains exactly one of the directed edges $(x, y)$ and $(y, x)$, and $\Gamma$ is connected if and only if $\Gamma_{i}$ is connected for each $i=1,2$. Furthermore, $A=\operatorname{Aut}\left(\Gamma_{i}\right)$ and $\Gamma_{i}$ is $A$-edge-transitive. In what follows we denote by $\vec{\Gamma}$ one of the digraphs $\Gamma_{1}$ and $\Gamma_{2}$.

Let $\Gamma$ be a half-arc-transitive graph of order $p^{3}$ for a prime $p$. Since there exists no half-arc-transitive graph of order less than 27 (see [1]), we have $p \geq 3$. By Proposition 2.3, $\Gamma=\operatorname{Cay}(G, S)$ and $\vec{\Gamma}=\operatorname{Cay}(G, R)$. Since a group of order $p$ or $p^{2}$ is abelian and there is no half-arc-transitive Cayley graph on an abelian group by Proposition 2.4, $\Gamma$ is connected, and so $G=\langle R\rangle$ and $S=R \cup R^{-1}$. Furthermore, $G=G_{1}(p)$ or $G_{2}(p)$, where

$$
\begin{aligned}
& G_{1}(p)=\left\langle a, b \mid a^{p^{2}}=1, b^{p}=1, b^{-1} a b=a^{1+p}\right\rangle, \\
& G_{2}(p)=\left\langle a, b, c \mid a^{p}=b^{p}=c^{p}=1,[a, b]=c,[a, c]=[b, c]=1\right\rangle .
\end{aligned}
$$

Since $G=\langle R\rangle$ is non-abelian, $R$ contains two elements $x$ and $y$ such that $x y \neq y x$, and since $|G|=p^{3}$, we have $\langle x, y\rangle=G$. For $G=G_{2}(p), x$ and $y$ have the same relations as do $a$ and $b$, which implies that we may assume that $a, b \in R$. Similarly, for $G=G_{1}(p)$ we may assume that $a \in R$. Thus we have the following observation:

Observation 3.1. Let $\Gamma$ be a half-arc-transitive graph of order $p^{3}$ for a prime $p$. Then $\Gamma=\operatorname{Cay}(G, S)$ and $\vec{\Gamma}=\operatorname{Cay}(G, R)$, where $G=G_{1}(p)$ or $G_{2}(p)$ with $p \geq 3, G=\langle R\rangle$ and $S=R \cup R^{-1}$. Furthermore,

(1) if $G=G_{1}(p)$ then $a \in R$;

(2) if $G=G_{2}(p)$ then $a, b \in R$.

Let us begin by considering normal half-arc-transitive Cayley graphs on $G_{2}(p)$ of valency 8. Since $G_{2}(p)$ has center $\langle c\rangle$, Proposition 2.1 implies $b^{j} a^{i}=a^{i} b^{j} c^{-i j}$ and $\left(a^{i} b^{j}\right)^{k}=a^{k i} b^{k j} c^{-2^{-1} k(k-1) i j}$ for $i, j, k \in \mathbb{Z}_{p}$. Our proofs will constantly be relying on these facts. 
Lemma 3.2. Let $\Gamma=\operatorname{Cay}\left(G_{2}(p), S\right)$ be a Cayley graph of valency 8. Then $\Gamma$ is normal and half-arc-transitive if and only if $4 \mid(p-1)$ and $\Gamma \cong \Gamma_{4, k}(p)$ for some $k$.

Proof. Let $\Gamma=\operatorname{Cay}\left(G_{2}(p), S\right)$ be normal and half-arc-transitive. Set $A=\operatorname{Aut}(\Gamma)$. By Observation 3.1, we have $\vec{\Gamma}=\operatorname{Cay}\left(G_{2}(p), R\right)$ with $p \geq 3, G_{2}(p)=\langle R\rangle$ and $S=$ $R \cup R^{-1}$. We may further assume $a, b, a^{i} b^{j} c^{k} \in R$. Since $\Gamma$ has valency 8 , we have $|S|=8$ and $|R|=4$. Since $\Gamma$ is normal, Proposition 2.6 implies that $A_{1}=\operatorname{Aut}\left(G_{2}(p), S\right)=$ $\operatorname{Aut}\left(G_{2}(p), R\right)$, which is transitive on $R$. Since $|R|=4$, Aut $\left(G_{2}(p), R\right) \leq S_{4}$. Thus, $\operatorname{Aut}\left(G_{2}(p), R\right)$ has a regular subgroup $M$ on $R$ such that $M \cong \mathbb{Z}_{2} \times \mathbb{Z}_{2}$ or $\mathbb{Z}_{4}$.

Case 1: $M \cong \mathbb{Z}_{2} \times \mathbb{Z}_{2}$. Let $\alpha_{1}, \alpha_{2} \in \operatorname{Aut}\left(G_{2}(p), R\right)$ and $M=\left\langle\alpha_{1}\right\rangle \times\left\langle\alpha_{2}\right\rangle \cong$ $\mathbb{Z}_{2} \times \mathbb{Z}_{2}$. Without loss of generality, we may assume that $a^{\alpha_{1}}=b$ and $b^{\alpha_{1}}=a$, and so $c^{\alpha_{1}}=c^{-1}$. This yields that $R=\left\{a, b, a^{i} b^{j} c^{k},\left(a^{i} b^{j} c^{k}\right)^{\alpha_{1}}\right\}=\left\{a, b, a^{i} b^{j} c^{k}, a^{j} b^{i} c^{-i j-k}\right\}$. Since $\left\langle\alpha_{1}, \alpha_{2}\right\rangle \cong \mathbb{Z}_{2} \times \mathbb{Z}_{2}$, we may assume that $a^{\alpha_{2}}=a^{i} b^{j} c^{k}$ and $\left(a^{i} b^{j} c^{k}\right)^{\alpha_{2}}=a$. Then $b^{\alpha_{2}}=a^{j} b^{i} c^{-i j-k}$ and so $c^{\alpha_{2}}=c^{i^{2}-j^{2}}$. By Proposition 2.1,

$$
\begin{aligned}
a=\left(a^{i} b^{j} c^{k}\right)^{\alpha_{2}} & =\left(a^{i} b^{j} c^{k}\right)^{i}\left(a^{j} b^{i} c^{-i j-k}\right)^{j}\left(c^{i^{2}-j^{2}}\right)^{k}=\left(a^{i} b^{j}\right)^{i}\left(a^{j} b^{i}\right)^{j} c^{k\left(i^{2}-j^{2}+i-j\right)-i j^{2}} \\
& =a^{i^{2}} b^{i j} c^{-2^{-1} i^{2} j(i-1)} a^{j^{2}} b^{i j} c^{-2^{-1} i j^{2}(j-1)} c^{k\left(i^{2}-j^{2}+i-j\right)-i j^{2}} \\
& =a^{i^{2}+j^{2}} b^{2 i j} c^{-i j^{3}+k\left(i^{2}-j^{2}+i-j\right)-i j^{2}-2^{-1} i^{2} j(i-1)-2^{-1} i j^{2}(j-1)}
\end{aligned}
$$

implying the following equations:

$$
\begin{aligned}
i^{2}+j^{2} & =1 ; \\
2 i j & =0 \\
-i j^{3}+k\left(i^{2}-j^{2}+i-j\right)-i j^{2}-2^{-1} i^{2} j(i-1)-2^{-1} i j^{2}(j-1) & =0 .
\end{aligned}
$$

As above, in what follows all equations are considered in $\mathbb{Z}_{p}$, unless otherwise stated. Since $\alpha_{1}$ interchanges $a$ and $b$, we can assume $i=0$ by Eq. (3.2), and so $j= \pm 1$ by Eq. (3.1). If $j=-1$ then $S=\left\{a, b, a^{-1} c^{-k}, b^{-1} c^{k}\right\} \cup\left\{a^{-1}, b^{-1}, a c^{k}, b c^{-k}\right\}$, and the automorphism of $G_{2}(p)$ induced by $a \mapsto a^{-1}, b \mapsto b c^{-k}, c \mapsto c^{-1}$, fixes $S$ setwise, contrary to Proposition 2.4. If $j=1$ then $k=0$ by Eq. (3.3), implying that $a^{i} b^{j} c^{k}=b$, a contradiction.

Case 2: $M \cong \mathbb{Z}_{4}$. Let $\alpha \in \operatorname{Aut}\left(G_{2}(p), R\right)$ and $M=\langle\alpha\rangle \cong \mathbb{Z}_{4}$. Then $R=$ $\left\{a, a^{\alpha}, a^{\alpha^{2}}, a^{\alpha^{3}}\right\}$, and since $G_{2}(p)=\langle R\rangle$, we have $\left\langle a, a^{\alpha}\right\rangle=G_{2}(p)$ and so $a \mapsto a$, $b \mapsto a^{\alpha}$ induces an automorphism of $G_{2}(p)$. We thus assume that $a^{\alpha}=b$, and $\alpha$ is induced by $a \mapsto b, b \mapsto a^{i} b^{j} c^{k}, c \mapsto c^{-i}$. It follows that

$$
R=\left\{a, b, a^{i} b^{j} c^{k}, a^{i j} b^{i+j^{2}} c^{-i^{2} j+k(j-i)-2^{-1} i j^{2}(j-1)}\right\} .
$$

Since

$$
\begin{aligned}
a=a^{\alpha^{4}} & =a^{i\left(i+j^{2}\right)} b^{j\left(2 i+j^{2}\right)} c^{i^{3} j+\left(k-i^{2} j\right)\left(i+j^{2}\right)-i k(j-i)+2^{-1} i^{2} j^{2}(j-1)-2^{-1} i j\left(i+j^{2}\right)\left(i+j^{2}-1\right)} \\
& =a^{i\left(i+j^{2}\right)} b^{j\left(2 i+j^{2}\right)} c^{k\left(i^{2}+j^{2}+i-i j\right)-i^{2} j^{3}+2^{-1} i j\left[i j^{2}-i j-\left(i+j^{2}\right)\left(i+j^{2}-1\right)\right]},
\end{aligned}
$$

we have the following equations:

$$
\begin{aligned}
i\left(i+j^{2}\right) & =1 ; \\
j\left(2 i+j^{2}\right) & =0 ; \\
k\left(i^{2}+j^{2}+i-i j\right)-i^{2} j^{3}+2^{-1} i j\left[i j^{2}-i j-\left(i+j^{2}\right)\left(i+j^{2}-1\right)\right] & =0 .
\end{aligned}
$$


By Eq. (3.5), either $j=0$ or $2 i+j^{2}=0$.

Case 2.1: $j=0$. By Eq. (3.4), $i= \pm 1$. If $i=1$ then $k=0$ by Eq. (3.6), and hence $a^{i} b^{j} c^{k}=a$, a contradiction. If $i=-1$ then $S=\left\{a, b, a^{-1} c^{k}, b^{-1} c^{k}\right\} \cup$ $\left\{a^{-1}, b^{-1}, a c^{-k}, b c^{-k}\right\}$, and the automorphism of $G_{2}(p)$ induced by $a \mapsto a^{-1}, b \mapsto b c^{-k}$, $c \mapsto c^{-1}$, fixes $S$ setwise, contrary to Proposition 2.4.

Case 2.2: $2 i+j^{2}=0$. Clearly, $i+j^{2}=-i$. By Eq. (3.4), $i^{2}=-1$ and so $i j^{2}=$ $1-i^{2}=2$. Since $j^{2}=-2 i$, Eq. (3.6) implies $2 k(1+i+i j)=-i j(1+i+i j)$, and hence $2 k i(1+i+i j)=j(1+i+i j)$, implying $2 k i-j=0$ or $1+i+i j=0$.

Suppose $2 k i-j=0$. Then $i j=-2 k$ and $k=-2^{-1} i j$. Since $i j^{2}=2$, we have $k=$ $-j^{-1}$ and $k(j-i)+1=-k(j+i)-1$. It follows that $S=\left\{a, b, a^{i} b^{j} c^{k}, a^{-2 k} b^{-i} c^{k(j-i)+1}\right\}$ $\cup\left\{a^{-1}, b^{-1}, a^{-i} b^{-j} c^{k}, a^{2 k} b^{i} c^{-k(j+i)-1}\right\}$. The automorphism of $G_{2}(p)$ induced by $a \mapsto$ $a^{-1}, b \mapsto b^{-1}, c \mapsto c$, fixes $S$ setwise, contrary to Proposition 2.4.

Thus, $1+i+i j=0$ and so $j=i-1$. Then $S=\left\{a, b, a^{i} b^{i-1} c^{k}, a^{-i-1} b^{-i} c^{1-k}\right\} \cup$ $\left\{a^{-1}, b^{-1}, a^{-i} b^{1-i} c^{-k+1+i}, a^{i+1} b^{i} c^{k-i}\right\}$. If $k=2^{-1}(1+i)$, then $k=-k+1+i$ and $1-k=k-i$, and hence the automorphism of $G_{2}(p)$ induced by $a \mapsto a^{-1}, b \mapsto b^{-1}$, $c \mapsto c$, fixes $S$ setwise, contrary to Proposition 2.4. Hence $k \neq 2^{-1}(1+i)$. Note that $i^{2}=-1$ implies that $4 \mid(p-1)$ and $i=\lambda$ is an element of order 4 in $\mathbb{Z}_{p}^{*}$. Then $j=\lambda-1$ and $k \neq 2^{-1}(1+\lambda)$. By the definition of $\Gamma_{4, k}(p)$ before Theorem 1.1, $\Gamma \cong \Gamma_{4, k}(p)$.

To finish the proof, we only need to show that $\Gamma_{4, k}(p)=\operatorname{Cay}\left(G_{2}(p), S_{4, k}\right)$ is normal and half-arc-transitive. Note that $S_{4, k}=\left\{a, a^{-1}, b, b^{-1}, a^{\lambda} b^{\lambda-1} c^{k}, a^{-\lambda} b^{1-\lambda} c^{-k+\lambda+1}\right.$, $\left.a^{-\lambda-1} b^{-\lambda} c^{1-k}, a^{\lambda+1} b^{\lambda} c^{k-\lambda}\right\}, \lambda$ is an element of order 4 in $\mathbb{Z}_{p}^{*}$, and $k \neq 2^{-1}(1+\lambda)$. Let $A=\operatorname{Aut}\left(\Gamma_{4, k}(p)\right)$ and set $R_{4, k}=\left\{a, b, a^{\lambda} b^{\lambda-1} c^{k}, a^{-\lambda-1} b^{-\lambda} c^{1-k}\right\}$. Then $S_{4, k}=R_{4, k} \cup$ $R_{4, k}^{-1}$. Let $\alpha$ be the automorphism of $G_{2}(p)$ induced by $a \mapsto b, b \mapsto a^{\lambda} b^{\lambda-1} c^{k}, c \mapsto c^{-\lambda}$. By Proposition 2.1, $\left(a^{\lambda} b^{\lambda-1} c^{k}\right)^{\alpha}=a^{-\lambda-1} b^{-\lambda} c^{1-k}$ and $\left(a^{-\lambda-1} b^{-\lambda} c^{1-k}\right)^{\alpha}=a$. Thus, $\alpha \in \operatorname{Aut}\left(G_{2}(p), S_{4, k}\right)$ has order 4 and permutes the elements of $R_{4, k}$ cyclically, implying that $\hat{G}_{2}(p) \rtimes\langle\alpha\rangle$ is half-arc-transitive on $\Gamma_{4, k}(p)$. To prove the normality and the half-arctransitivity of $\Gamma_{4, k}(p)$, it suffices to show that $A=\hat{G}_{2}(p) \rtimes\langle\alpha\rangle$.

Write $L=\operatorname{Aut}\left(G_{2}(p), S_{4, k}\right)$. Then $L$ acts on $S_{4, k}$ faithfully. Set $\Omega_{1}=\left\{a, a^{-1}\right\}, \Omega_{2}=$ $\left\{b, b^{-1}\right\}, \Omega_{3}=\left\{a^{\lambda} b^{\lambda-1} c^{k}, a^{-\lambda} b^{1-\lambda} c^{-k+\lambda+1}\right\}, \Omega_{4}=\left\{a^{-\lambda-1} b^{-\lambda} c^{1-k}, a^{\lambda+1} b^{\lambda} c^{k-\lambda}\right\}$. Since $L \leq \operatorname{Aut}\left(G_{2}(p)\right),\left\{\Omega_{1}, \Omega_{2}, \Omega_{3}, \Omega_{4}\right\}$ is a complete imprimitive block system of $L$ on $S_{4, k}$. Let $\Omega=\left\{\Omega_{1}, \Omega_{2}, \Omega_{3}, \Omega_{4}\right\}$. Since $\alpha \in L, L$ is transitive on $\Omega$.

Claim: $L_{a}=1$ and $L_{x}=1$ for any $x \in S_{4, k}$. Let $\beta \in L_{a}$. Then $a^{\beta}=a$ and $\Omega_{1}^{\beta}=\Omega_{1}$. Thus, $\left(\Omega_{2} \cup \Omega_{3} \cup \Omega_{4}\right)^{\beta}=\Omega_{2} \cup \Omega_{3} \cup \Omega_{4}$, and so $b^{\beta} \in \Omega_{2} \cup \Omega_{3} \cup \Omega_{4}$, that is, $b^{\beta}=b$, $b^{-1}, a^{\lambda} b^{\lambda-1} c^{k}, a^{-\lambda} b^{1-\lambda} c^{-k+\lambda+1}, a^{-\lambda-1} b^{-\lambda} c^{1-k}$ or $a^{\lambda+1} b^{\lambda} c^{k-\lambda}$. As $\lambda$ is an element of order 4 in $\mathbb{Z}_{p}^{*}$, we have $\lambda \neq 0, \pm 1$. If $b^{\beta}=b^{-1} \in \Omega_{2}$ then $c^{\beta}=c^{-1}$ and $\Omega_{2}^{\beta}=\Omega_{2}$. It follows that $\left(\Omega_{3} \cup \Omega_{4}\right)^{\beta}=\Omega_{3} \cup \Omega_{4}$, implying that $\left(a^{\lambda} b^{\lambda-1} c^{k}\right)^{\beta}=a^{\lambda} b^{1-\lambda} c^{-k} \in \Omega_{3} \cup \Omega_{4}$, which is impossible. If $b^{\beta}=a^{\lambda} b^{\lambda-1} c^{k} \in \Omega_{3}$ then $c^{\beta}=c^{\lambda-1}$ and $\Omega_{2}^{\beta}=\Omega_{3}$. Thus, $\Omega_{3}^{\beta} \subseteq \Omega_{2} \cup \Omega_{4}$, but $\left(a^{\lambda} b^{\lambda-1} c^{k}\right)^{\beta}=a^{-1} b^{-2 \lambda} c^{-\lambda+2+2 k(\lambda-1)} \notin \Omega_{2} \cup \Omega_{4}$, a contradiction. If $b^{\beta}=a^{-\lambda} b^{1-\lambda} c^{-k+\lambda+1} \in \Omega_{3}$, then $c^{\beta}=c^{1-\lambda}$ and $\Omega_{2}^{\beta}=\Omega_{3}$. Thus, $\Omega_{3}^{\beta} \subseteq \Omega_{2} \cup \Omega_{4}$ and $\left(a^{\lambda} b^{\lambda-1} c^{k}\right)^{\beta}=a^{2 \lambda+1} b^{2 \lambda} c^{-\lambda-2 k(\lambda-1)}$ implies that $\left(a^{\lambda} b^{\lambda-1} c^{k}\right)^{\beta}=b^{-1}$. It follows that $\Omega_{3}^{\beta}=\Omega_{2}$ and so $\Omega_{4}^{\beta}=\Omega_{4}$, which is impossible because $\left(a^{\lambda+1} b^{\lambda} c^{k-\lambda}\right)^{\beta}=$ $a^{\lambda+2} b^{\lambda+1} c^{-2 k \lambda+k-\lambda-2} \notin \Omega_{4}$. If $b^{\beta}=a^{-\lambda-1} b^{-\lambda} c^{1-k} \in \Omega_{4}$, then $c^{\beta}=c^{-\lambda}$ and $\Omega_{2}^{\beta}=\Omega_{4}$. Thus, $\Omega_{3}^{\beta} \subseteq \Omega_{2} \cup \Omega_{3}$ and $\left(a^{\lambda} b^{\lambda-1} c^{k}\right)^{\beta}=a^{\lambda+2} b^{\lambda+1} c^{-2 k \lambda+k-\lambda-2}$ implies that $\left(a^{\lambda} b^{\lambda-1} c^{k}\right)^{\beta}=b^{-1}$ and $\lambda=-2$. It follows that $\Omega_{3}^{\beta}=\Omega_{2}$ and so $\Omega_{4}^{\beta}=\Omega_{3}$. This forces $\lambda=2$ as $\left(a^{\lambda+1} b^{\lambda} c^{k-\lambda}\right)^{\beta}=a^{2} b c^{-2 k \lambda+\lambda-2} \in \Omega_{3}$, and hence $\lambda=2=-2$, a contradiction. If $b^{\beta}=a^{\lambda+1} b^{\lambda} c^{k-\lambda} \in \Omega_{4}$ then $c^{\beta}=c^{\lambda}$ and $\Omega_{2}^{\beta}=\Omega_{4}$. Thus, $\Omega_{3}^{\beta} \subseteq \Omega_{2} \cup \Omega_{3}$ 
and so $\left(a^{\lambda} b^{\lambda-1} c^{k}\right)^{\beta}=a^{\lambda-2} b^{-\lambda-1} c^{2 k \lambda-k-\lambda} \in \Omega_{2} \cup \Omega_{3}$, which is impossible. The above arguments mean that $b^{\beta}=b$, implying $\beta=1$. Thus, $L_{a}=1$, and since $\Omega_{1}$ is a block of $L$, we have $L_{a^{-1}} \leq L_{a}=1$. The transitivity of $\langle\alpha\rangle$ on $\Omega$ implies $L_{x}=1$ for any $x \in S_{4, k}$, as claimed.

Let $K$ be the kernel of $L$ on $\Omega$. Then $K$ fixes each $\Omega_{i}$ setwise, and by Claim, $|K|=$ $\left|K_{a}\right|\left|a^{K}\right| \leq 2$. Suppose $|K|=2$. Then the unique involution, say $\gamma$, in $K$ interchanges the two elements in each $\Omega_{i}$ because $L_{x}=1$. In particular, $\gamma$ is induced by $a^{\gamma}=a^{-1}, b^{\gamma}=$ $b^{-1}$ and $c^{\gamma}=c$. It follows that $\left(a^{\lambda} b^{\lambda-1} c^{k}\right)^{\gamma}=a^{-\lambda} b^{1-\lambda} c^{k}$, and since $\left(a^{\lambda} b^{\lambda-1} c^{k}\right)^{\gamma} \in \Omega_{3}$, we have $a^{-\lambda} b^{1-\lambda} c^{k}=a^{-\lambda} b^{1-\lambda} c^{-k+\lambda+1}$, forcing that $k=2^{-1}(1+\lambda)$, a contradiction. Thus, $K=1$ and $L \leq S_{4}$, the symmetric group of degree 4 .

Since $L_{x}=1$ for any $x \in S_{4, k}, L$ is semiregular on $S_{4, k}$, and so $|L|$ is a divisor of 8. Since $\alpha \in L$, we have $|L|=4$ or 8 . Suppose $|L|=8$. Since $L \leq S_{4}, L$ is the dihedral group of order 8 , and so $\alpha^{2} \in Z(L)$. Note that $\alpha^{2}$ interchanges $\Omega_{1}$ and $\Omega_{3}$, and $\Omega_{2}$ and $\Omega_{4}$. Then $L_{\Omega_{1}}=L_{\Omega_{1}}^{\alpha^{2}}=L_{\Omega_{3}}$. Since $L$ is transitive on $\Omega,\left|L_{\Omega_{1}}\right|=2$. Let $\delta$ be the unique involution in $L_{\Omega_{1}}$. Then $\Omega_{1}^{\delta}=\Omega_{1}$ and $\Omega_{3}^{\delta}=\Omega_{3}$. Since $L_{a}=1$, we have $a^{\delta}=a^{-1}$, and since $K=1$, we have $\Omega_{2}^{\delta}=\Omega_{4}$. On the other hand, $\langle\alpha\rangle \unlhd L$ and so $R_{4, k}$ is an imprimitive block of $L$, yielding $R_{4, k}^{\delta}=R_{4, k}^{-1}$. It follows that $b^{\delta} \in \Omega_{2}^{\delta} \cap R_{4, k}^{\delta}=$ $\Omega_{4} \cap R_{4, k}^{-1}$, that is, $b^{\delta}=a^{\lambda+1} b^{\lambda} c^{k-\lambda}$. Thus, $c^{\delta}=c^{-\lambda}$, and since $\delta$ is an involution, $b=\left(a^{\lambda+1} b^{\lambda} c^{k-\lambda}\right)^{\delta}=a^{-2} b^{-1} c^{-1}$, which is impossible. Thus, $|L|=4$ and $L=\langle\alpha\rangle$. Clearly, $p \nmid|L|=\left|\operatorname{Aut}\left(G_{2}(p), S_{4, k}\right)\right|$. By Proposition 2.7, $\Gamma_{4, k}(p)$ is a normal Cayley graph, and by Proposition 2.6, $A=\hat{G}_{2}(p) \rtimes\langle\alpha\rangle$.

Remark 3.3. The above proof implies $\operatorname{Aut}\left(\Gamma_{4, k}(p)\right)=\hat{G}_{2}(p) \rtimes\langle\alpha\rangle$ and $\operatorname{Aut}\left(\Gamma_{4, k}(p)\right)_{1}=$ $\operatorname{Aut}\left(G_{2}(p), S_{4, k}\right)=\langle\alpha\rangle$, where $\alpha$ is the automorphism of $G_{2}(p)$ of order 4 induced by $a \mapsto$ $b$ and $b \mapsto a^{\lambda} b^{\lambda-1} c^{k}$. Moreover, the automorphism $\alpha$ cyclically permutes the elements in $\left\{a, b, a^{\lambda} b^{\lambda-1} c^{k}, a^{-\lambda-1} b^{-\lambda} c^{1-k}\right\}$.

Lemma 3.4. There are exactly $(p-1) / 2$ nonisomorphic graphs of the form $\Gamma_{4, k}(p)$.

Proof. By definition, $S_{4, k}=\left\{a, a^{-1}, b, b^{-1}, a^{\lambda} b^{\lambda-1} c^{k}, a^{-\lambda} b^{1-\lambda} c^{\lambda-k+1}, a^{-\lambda-1} b^{-\lambda} c^{1-k}\right.$, $\left.a^{\lambda+1} b^{\lambda} c^{k-\lambda}\right\}$ and $\Gamma_{4, k}(p)=\operatorname{Cay}\left(G_{2}(p), S_{4, k}\right)$, where $\lambda$ is an element of order 4 in $\mathbb{Z}_{p}^{*}$, $k \in \mathbb{Z}_{p}$ with $k \neq 2^{-1}(1+\lambda)$. Thus, $4 \mid(p-1)$. Since $\Gamma_{4, k}(p)$ does not depend on the choice of $\lambda$ (see the paragraph before Theorem 1.1), there are at most $p-1$ nonisomorphic half-arc-transitive graphs of the form $\Gamma_{4, k}(p)\left(k \neq 2^{-1}(1+\lambda)\right)$. To finish the proof, it suffices to show that $\Gamma_{4, k}(p) \cong \Gamma_{4, l}(p)\left(k, l \neq 2^{-1}(1+\lambda)\right)$ if and only if $l=k$ or $l=1+\lambda-k$.

Let $l=1+\lambda-k$. One may easily show that the automorphism of $G_{2}(p)$ induced by $a \mapsto a^{-1}, b \mapsto b^{-1}, c \mapsto c$, maps $S_{4, k}$ to $S_{4,1+\lambda-k}=S_{4, l}$, and so $\Gamma_{4, k}(p) \cong \Gamma_{4, l}(p)$.

Let $\Gamma_{4, k}(p) \cong \Gamma_{4, l}(p)\left(k, l \neq 2^{-1}(1+\lambda)\right)$. Set

$$
R_{4, i}=\left\{a, b, a^{\lambda} b^{\lambda-1} c^{i}, a^{-\lambda-1} b^{-\lambda} c^{1-i}\right\} .
$$

Then $S_{4, k}=R_{4, k} \cup R_{4, k}^{-1}$ and $S_{4, l}=R_{4, l} \cup R_{4, l}^{-1}$. Since $4 \mid(p-1)$, we have $p \geq$ 5 , and by Proposition 2.5, there exists $\sigma \in \operatorname{Aut}\left(G_{2}(p)\right)$ such that $S_{4, k}^{\sigma}=S_{4, l}$. This implies that $\sigma$ maps the stabilizer $\operatorname{Aut}\left(\Gamma_{4, k}(p)\right)_{1}$ to the stabilizer $\operatorname{Aut}\left(\Gamma_{4, l}(p)\right)_{1}$. It follows $\operatorname{Aut}\left(G_{2}(p), S_{4, k}\right)^{\sigma}=\operatorname{Aut}\left(G_{2}(p), S_{4, l}\right)$ because $\operatorname{Aut}\left(\Gamma_{4, k}(p)\right)_{1}=\operatorname{Aut}\left(G_{2}(p), S_{4, k}\right)$ and $\operatorname{Aut}\left(\Gamma_{4, l}(p)\right)_{1}=\operatorname{Aut}\left(G_{2}(p), S_{4, l}\right)$ by Remark 3.3. Moreover, $\operatorname{Aut}\left(G_{2}(p), S_{4, k}\right)$ is regular on both $R_{4, k}$ and $R_{4, k}^{-1}$, and $\operatorname{Aut}\left(G_{2}(p), S_{4, l}\right)$ is regular on both $R_{4, l}$ and $R_{4, l}^{-1}$. 
Thus, $R_{4, k}^{\sigma}=R_{4, l}$ or $R_{4, l}^{-1}$, and replacing $\sigma$ by a multiplication of $\sigma$ and an element in $\operatorname{Aut}\left(G_{2}(p), S_{4, l}\right)$, we may assume that $a^{\sigma}=a$ if $R_{4, k}^{\sigma}=R_{4, l}$, and $a^{\sigma}=a^{-1}$ if $R_{4, k}^{\sigma}=R_{4, l}^{-1}$.

Assume $R_{4, k}^{\sigma}=R_{4, l}$ with $a^{\sigma}=a$. Then $b^{\sigma} \in R_{4, l}$ and $b^{\sigma}=b, a^{\lambda} b^{\lambda-1} c^{l}$ or $a^{-\lambda-1} b^{-\lambda} c^{1-l}$. If $b^{\sigma}=a^{\lambda} b^{\lambda-1} c^{l}$ then $c^{\sigma}=c^{\lambda-1}$. By Proposition 2.1, $\left(a^{\lambda} b^{\lambda-1} c^{k}\right)^{\sigma}=$ $a^{-1} b^{-2 \lambda} c^{-\lambda+2+(k+l)(\lambda-1)} \in R_{4, l}$, which is impossible. If $b^{\sigma}=a^{-\lambda-1} b^{-\lambda} c^{1-l}$ then $c^{\sigma}=c^{-\lambda}$, and hence $\left(a^{\lambda} b^{\lambda-1} c^{k}\right)^{\sigma}=a^{\lambda+2} b^{\lambda+1} c^{-\lambda-2-l(\lambda-1)-k \lambda} \in R_{4, l}$, which is impossible. If $b^{\sigma}=b$ then $c^{\sigma}=c$, and hence $\left(a^{\lambda} b^{\lambda-1} c^{k}\right)^{\sigma}=a^{\lambda} b^{\lambda-1} c^{k} \in R_{4, l}$, implying that $l=k$.

Assume $R_{4, k}^{\sigma}=R_{4, l}^{-1}$ with $a^{\sigma}=a^{-1}$. Then $b^{\sigma} \in R_{4, l}^{-1}$, and $b^{\sigma}=b^{-1}, a^{-\lambda} b^{1-\lambda} c^{-l+\lambda+1}$ or $a^{\lambda+1} b^{\lambda} c^{l-\lambda}$. If $b^{\sigma}=a^{-\lambda} b^{1-\lambda} c^{-l+\lambda+1}$ then $c^{\sigma}=c^{\lambda-1}$. By Proposition 2.1, we have $\left(a^{\lambda} b^{\lambda-1} c^{k}\right)^{\sigma}=a b^{2 \lambda} c^{-\lambda+(k-l)(\lambda-1)} \in R_{4, l}^{-1}$, which is impossible. If $b^{\sigma}=a^{\lambda+1} b^{\lambda} c^{l-\lambda}$ then $c^{\sigma}=c^{-\lambda}$ and hence $\left(a^{\lambda} b^{\lambda-1} c^{k}\right)^{\sigma}=a^{-\lambda-2} b^{-\lambda-1} c^{-\lambda-k \lambda+l(\lambda-1)} \in R_{4, l}^{-1}$, which is impossible. If $b^{\sigma}=b^{-1}$ then we have $c^{\sigma}=c$ and $\left(a^{\lambda} b^{\lambda-1} c^{k}\right)^{\sigma}=a^{-\lambda} b^{1-\lambda} c^{k} \in R_{4, l}^{-1}$, implying that $l=1+\lambda-k$.

By Magma [4], a brute force computer search can be performed to verify the following lemma, but we have also verified the correctness of the lemma theoretically. Since the proof is rather long, we will not present it in the paper but are willing to provide it upon request (also see [29]).

Lemma 3.5. There is no half-arc-transitive graph of order 27 and valency 6 or 8.

Now we are ready to prove Theorem 1.1 .

Proof of Theorem 1.1. Let $\Gamma$ be a half-arc-transitive graph of order $p^{3}$ and valency 6 or 8, and let $A=\operatorname{Aut}(\Gamma)$. By Observation 3.1, $\Gamma=\operatorname{Cay}(G, S)$ and $\vec{\Gamma}=\operatorname{Cay}(G, R)$ for some group $G=G_{1}(p)$ or $G_{2}(p)$ with $p \geq 3$, where $G=\langle R\rangle$ and $S=R \cup R^{-1}$. By Lemma 3.5, $p \geq 5$, and by the half-arc-transitivity of $\Gamma, A=\operatorname{Aut}(\vec{\Gamma})$ and $A_{1}$ is transitive on $R$. Since $G=\langle R\rangle$, Aut $(G, R)$ acts faithfully on $R$, and since $|R|<5$, $\operatorname{Aut}(G, R)$ is a $p^{\prime}$-group. Since $G$ is a non-abelian group of order $p^{3}, G$ is 2 -genetic, that is, each normal subgroup of $G$ can be generated by two elements. By Proposition $2.7, \vec{\Gamma}$ is normal, and by Proposition 2.6, $A_{1}=\operatorname{Aut}(G, R)$. Since $A=\operatorname{Aut}(\Gamma)=\operatorname{Aut}(\vec{\Gamma})$, we have $A_{1}=\operatorname{Aut}(G, S)$, and so $\Gamma$ is normal.

The theorem is true for $G=G_{1}(p)$ by Proposition 2.2. Now assume $G=G_{2}(p)$. If $\Gamma$ has valency 8 , the theorem is also true by Lemma 3.2. We may thus assume that $\Gamma$ has valency 6 , that is, $|R|=3$. We prove that this is not possible.

By Observation 3.1, $R=\left\{a, b, a^{i} b^{j} c^{k}\right\}$, where $i, j, k \in \mathbb{Z}_{p}$. Since $\operatorname{Aut}(G, R)$ is transitive on $R$, there exists $\alpha \in \operatorname{Aut}\left(G_{2}(p)\right)$ of order 3 permuting the elements in $R$ cyclically. If necessary, replace $\alpha$ by $\alpha^{2}$, and then we may assume that $\alpha$ is induced by $a \mapsto b, b \mapsto a^{i} b^{j} c^{k}$, and then $c \mapsto c^{-i}$ by Proposition 2.1. Thus, $a=\left(a^{i} b^{j} c^{k}\right)^{\alpha}=$ $a^{i j} b^{i+j^{2}} c^{-i^{2} j-2^{-1} i j^{2}(j-1)+k(j-i)}$, and so we have:

$$
\begin{aligned}
i j & =1 ; \\
i+j^{2} & =0 ; \\
-i^{2} j+k(j-i)-2^{-1} i j^{2}(j-1) & =0 .
\end{aligned}
$$


By Eqs. (3.7) and (3.8), $j^{3}+1=0$, implying $(j+1)\left(j^{2}-j+1\right)=0$. Thus, either $j+1=0$ or $j^{2}-j+1=0$. If $j+1=0$ then $j=-1$. By Eq. (3.8), $i=-1$ and so $S=\left\{a, b, a^{-1} b^{-1} c^{k}\right\} \cup\left\{a^{-1}, b^{-1}, a b c^{-1-k}\right\}$, but the automorphism of $G$ induced by $a \mapsto a^{-1}, b \mapsto a b c^{-1-k}, c \mapsto c^{-1}$, fixes $S$ setwise, contrary to Proposition 2.4. If $j^{2}-j+1=0$ then by Eq. (3.8), $i=1-j$, and since $i j=1$, Eq. (3.9) implies that $-i+k(j-i)-2^{-1} j(j-1)=j-1+k(j+j-1)+2^{-1}=(2 j-1)\left(k+2^{-1}\right)=0$. It follows that either $2 j-1=0$ or $k+2^{-1}=0$. For $2 j-1=0$, we have $j=2^{-1}$ and $i=1-j=1-2^{-1}=2^{-1}$, but then Eq. (3.7) implies $4=1$ in $\mathbb{Z}_{p}$, contradicting $p \neq 3$. For $k+2^{-1}=0$, we have $S=\left\{a, b, a^{1-j} b^{j} c^{-2^{-1}}\right\} \cup\left\{a^{-1}, b^{-1}, a^{j-1} b^{-j} c^{-2^{-1}}\right\}$, and the automorphism of $G$ induced by $a \mapsto a^{-1}, b \mapsto b^{-1}, c \mapsto c$, fixes $S$ setwise, a contradiction.

\section{References}

[1] B. Alspach, D. Marušič and L. Nowitz, Constructing graphs which are 1/2-transitive, J. Austral. Math. Soc. Ser. A 56 (1994), 391-402, doi:10.1023/a:1022466626755.

[2] B. Alspach and M. Y. Xu, 1/2-transitive graphs of order 3p, J. Algebraic Combin. 3 (1994), 347-355, doi:10.1023/a:1022466626755.

[3] I. Antončič and P. Šparl, Classification of quartic half-arc-transitive weak metacirculants of girth at most 4, Discrete Math. 339 (2016), 931-945, doi:10.1016/j.disc.2015.10.015.

[4] W. Bosma, J. Cannon and C. Playoust, The Magma algebra system I: the user language, J. Symbolic Comput. 24 (1997), 235-265, doi:10.1006/jsco.1996.0125.

[5] I. Z. Bouwer, Vertex and edge transitive but not 1-transitive graphs, Canad. Math. Bull. 13 (1970), 231-237, doi:10.4153/cmb-1970-047-8.

[6] Y. Cheng and J. Oxley, On weakly symmetric graphs of order twice a prime, J. Combin. Theory Ser. B 42 (1987), 196-211, doi:10.1016/0095-8956(87)90040-2.

[7] M. D. E. Conder and D. Marušič, A tetravalent half-arc-transitive graph with non-abelian vertex stabilizer, J. Combin. Theory Ser. B 88 (2003), 67-76, doi:10.1016/S0095-8956(02)00036-9.

[8] M. D. E. Conder, P. Potočnik and P. Šparl, Some recent discoveries about half-arc-transitive graphs, Ars Math. Contemp. 8 (2015), 149-162, http://amc-journal.eu/index. $\mathrm{php/amc/article/view/557.}$

[9] E. Dobson, Automorphism groups of metacirculant graphs of order a product of two distinct primes, Combin. Probab. Comput. 15 (2006), 105-130, doi:10.1017/S0963548305007066.

[10] Y.-Q. Feng, J. H. Kwak, X. Wang and J.-X. Zhou, Tetravalent half-arc-transitive graphs of order $p^{4}$, European J. Combin. 29 (2008), 555-567, doi:10.1016/j.ejc.2007.05.004.

[11] Y.-Q. Feng, J. H. Kwak, X. Wang and J.-X. Zhou, Tetravalent half-arc-transitive graphs of order 2pq, J. Algebraic Combin. 33 (2011), 543-553, doi:10.1007/s10801-010-0257-1.

[12] C. D. Godsil, On the full automorphism group of a graph, Combinatorica 1 (1981), 243-256, doi:10.1007/bf02579330.

[13] A. Hujdurović, K. Kutnar and D. Marušič, Half-arc-transitive group actions with a small number of alternets, J. Combin. Theory Ser. A 124 (2014), 114-129, doi:10.1016/j.jcta.2014.01.005.

[14] B. Huppert, Endliche Gruppen. I, Die Grundlehren der Mathematischen Wissenschaften, Band 134, Springer-Verlag, Berlin-New York, 1967, doi:10.1007/978-3-642-64981-3.

[15] K. Kutnar, D. Marušič and P. Šparl, An infinite family of half-arc-transitive graphs with universal reachability relation, European J. Combin. 31 (2010), 1725-1734, doi:10.1016/j.ejc.2010. 03.006 . 
[16] K. Kutnar, D. Marušič, P. Šparl, R.-J. Wang and M.-Y. Xu, Classification of half-arc-transitive graphs of order 4p, European J. Combin. 34 (2013), 1158-1176, doi:10.1016/j.ejc.2013.04. 004.

[17] C. H. Li, On isomorphisms of connected Cayley graphs, Discrete Math. 178 (1998), 109-122, doi:10.1016/s0012-365x(97)81821-3.

[18] C. H. Li and H.-S. Sim, On half-transitive metacirculant graphs of prime-power order, J. Combin. Theory Ser. B 81 (2001), 45-57, doi:10.1006/jctb.2000.1992.

[19] D. Marušič, Vertex transitive graphs and digraphs of order $p^{k}$, in: Cycles in graphs (Burnaby, B.C., 1982), North-Holland, Amsterdam, volume 115 of North-Holland Math. Stud., pp. 115128, 1985, doi:10.1016/s0304-0208(08)73001-9.

[20] D. Marušič, Half-transitive group actions on finite graphs of valency 4, J. Combin. Theory Ser. B 73 (1998), 41-76, doi:10.1006/jctb.1997.1807.

[21] D. Marušič, Quartic half-arc-transitive graphs with large vertex stabilizers, Discrete Math. 299 (2005), 180-193, doi:10.1016/j.disc.2004.02.025.

[22] D. Marušič and P. Šparl, On quartic half-arc-transitive metacirculants, J. Algebraic Combin. 28 (2008), 365-395, doi:10.1007/s10801-007-0107-y.

[23] P. Potočnik, P. Spiga and G. Verret, A census of 4-valent half-arc-transitive graphs and arc-transitive digraphs of valence two, Ars Math. Contemp. 8 (2015), 133-148, http: //amc-journal.eu/index.php/amc/article/view/559.

[24] D. J. S. Robinson, A Course in the Theory of Groups, volume 80 of Graduate Texts in Mathematics, Springer-Verlag, New York, 2nd edition, 1996, doi:10.1007/978-1-4419-8594-1.

[25] P. Spiga, Constructing half-arc-transitive graphs of valency four with prescribed vertex stabilizers, Graphs Combin. 32 (2016), 2135-2144, doi:10.1007/s00373-016-1678-y.

[26] W. T. Tutte, Connectivity in Graphs, Mathematical Expositions, No. 15, University of Toronto Press, Toronto, Ont.; Oxford University Press, London, 1966.

[27] P. Šparl, A classification of tightly attached half-arc-transitive graphs of valency 4, J. Combin. Theory Ser. B 98 (2008), 1076-1108, doi:10.1016/j.jctb.2008.01.001.

[28] P. Šparl, On the classification of quartic half-arc-transitive metacirculants, Discrete Math. 309 (2009), 2271-2283, doi:10.1016/j.disc.2008.05.006.

[29] Y. Wang and Y.-Q. Feng, Half-arc-transitive graphs of prime-cube order of small valencies, 2016, arXiv:1605.08123 [math.CO].

[30] Y. Wang, Y.-Q. Feng and J.-X. Zhou, Cayley digraphs of 2-genetic groups of odd prime-power order, J. Combin. Theory Ser. A 143 (2016), 88-106, doi:10.1016/j.jcta.2016.05.001.

[31] M.-Y. Xu, Half-transitive graphs of prime-cube order, J. Algebraic Combin. 1 (1992), 275-282, doi:10.1023/a:1022440002282.

[32] M.-Y. Xu, Automorphism groups and isomorphisms of Cayley digraphs, Discrete Math. 182 (1998), 309-319, doi:10.1016/s0012-365x(97)00152-0.

[33] J.-X. Zhou, Tetravalent half-arc-transitive p-graphs, J. Algebraic Combin. 44 (2016), 947-971, doi:10.1007/s10801-016-0696-4. 